\title{
Multimodal Evaluation of Presumed Tuberculous Serpiginous-Like Choroiditis
}

DOI:

10.1080/09273948.2018.1501497

\section{Document Version}

Accepted author manuscript

Link to publication record in Manchester Research Explorer

\section{Citation for published version (APA):}

Wang, H., Tan, S. Z., Aslam, T., Jones, N. P., \& Steeples, L. R. (2018). Multimodal Evaluation of Presumed Tuberculous Serpiginous-Like Choroiditis. Ocular Immunology and Inflammation, 1-5.

https://doi.org/10.1080/09273948.2018.1501497

\section{Published in:}

Ocular Immunology and Inflammation

\section{Citing this paper}

Please note that where the full-text provided on Manchester Research Explorer is the Author Accepted Manuscript or Proof version this may differ from the final Published version. If citing, it is advised that you check and use the publisher's definitive version.

\section{General rights}

Copyright and moral rights for the publications made accessible in the Research Explorer are retained by the authors and/or other copyright owners and it is a condition of accessing publications that users recognise and abide by the legal requirements associated with these rights.

\section{Takedown policy}

If you believe that this document breaches copyright please refer to the University of Manchester's Takedown Procedures [http://man.ac.uk/04Y6Bo] or contact uml.scholarlycommunications@manchester.ac.uk providing relevant details, so we can investigate your claim.

\section{OPEN ACCESS}


2

3 Haoyu Wang ${ }^{1,}$ MBChB; Shi Zhuan Tan ${ }^{1,2}$ FRCOphth; Tariq Aslam ${ }^{1,2}$ PhD, FRCSEd;

4 Nicholas P Jones ${ }^{1,2}$ FRCOphth ; Laura R Steeples ${ }^{1,2}$ FRCOphth

5

6 1. Centre for Ophthalmology and Vision Sciences, Faculty of Medical and Human Sciences,

7 University of Manchester, Manchester, UK; 2.Manchester Royal Eye Hospital, Manchester

8 University NHS Foundation Trust

9 Corresponding author: Dr Haoyu Wang

10 Email: derekhaoyuwang@gmail.com

11 Telephone: 01612761234

12 Address: Manchester Royal Eye Hospital, Manchester University NHS Foundation Trust,

13 Oxford Road, Manchester, UK, M13 9WL.

14

15 The authors have no conflict of interest and have not received any funding for the materials

16 reported in the article.

17

18 Key words: presumed tuberculous serpiginous-like choroiditis, optical coherence tomography

19 angiography, multimodal imaging, fundus autofluorescence, uveitis, swept-source optical 20 coherence tomography. 


\section{Abstract}

22 Purpose: To evaluate multimodal imaging findings in longitudinal follow-up of a patient with 23 presumed tuberculous serpiginous-like choroiditis (TB-SLC).

24 Method: We evaluated multimodal imaging in a 62-year-old male with TB-SLC. Correlation 25 between optical coherence tomography angiography (OCTA), swept-source OCT (SS-OCT) 26 and fundus autofluorescence (FAF) at defined disease stages and evolution of observed 27 imaging descriptors during long-term follow-up has not been previously reported.

28 Results: OCTA of the active lesion demonstrated defined areas of choriocapillaris 29 hypoperfusion, suggesting inflammatory vascular occlusive pathology. Over 9-month follow30 up, OCTA illustrated sequential improvement in choriocapillaris flow, suggesting vascular 31 remodelling. This correlated with progressive change in FAF signal and transition to diffuse 32 hypoautofluorescence. SS-OCT demonstrated focal choroidal thickening and retinal pigment 33 epithelium elevation in acute phase and resolution in time.

34 Conclusion: Multimodal imaging, particularly novel non-invasive technologies such as 35 OCTA and SS-OCT, improves our understanding of the pathogenesis and evolution of 36 disease in TB-SLC. 
Introduction

47 Serpiginous choroiditis (SC) is a rare chronic idiopathic inflammatory condition 48 characterised by a geographic lesion pattern with intermittent centrifugal spread with an 49 advancing active edge. Tuberculous choroiditis simulating SC is now recognised as a distinct 50 clinical entity known as tuberculous serpiginous like choroiditis (TB-SLC). ${ }^{1-4}$ Fundus 51 fluorescein angiography (FFA) and indocyanine green angiography (ICGA) are well52 established for in part assessing the retina and choroid in TB-SLC and defining active 53 disease. Fundus autofluorescence (FAF) classified disease stages and enhanced depth 54 imaging optical coherence tomography (EDI-OCT) signs have been described. ${ }^{5-7}$ OCT 55 angiography (OCTA) technology provides non-invasive vascular imaging at specific depths 56 including the retina plexus and choriocapillaris. The role of OCTA in long-term follow-up of 57 TB-SLC has not been previously evaluated. We report a case of TB-SLC and evolution of 58 multimodal imaging findings, including swept-source OCT (SS-OCT) and SS-OCTA, at 59 sequential disease stages.

60

\section{Case Report}

62 A 62 year-old Caucasian male presented with a 6-week history of photopsia and scotoma in 63 his right eye. Best-corrected visual acuity was 0.0 LogMAR in both eyes. The left eye was 64 normal. In the right eye a creamy yellow serpiginous-like subretinal lesion was seen in the 65 nasal peripapillary region contiguous with the optic disc (Figure 1A). The superior aspect of 66 the lesion was defined with a light surrounding border; the inferior portion demonstrated an 67 ill-defined edge and oedematous appearance, indicating active disease and centrifugal 68 extension.

69 Multimodal imaging demonstrated active disease (Figures 1-3). FAF revealed a 70 hyperautofluorescent lesion with rapid progression and contiguous extension from imaging 
71 performed in a different centre 4 weeks earlier (Figure 2A and B, Spectralis ${ }^{\circledR}$ Heidelberg

72 Engineering, Heidelberg, Germany). Surrounding ill-defined hyperautofluorescence was 73 evident (Figure 2A); this persisted at the active inferior edge but became a thin defined 74 border of hypoautofluorescence around the rest of the lesion at 1 month (Figure 2B) 75 suggesting transition towards inactivity. Fundus fluorescein angiography (FFA) and ICG 76 delineated the lesion and different disease stages within it: in the inferior portion early FFA 77 hypofluorescence was followed by late leakage indicating active disease contrasted by 78 hypofluorescence and subsequently a bright hyperfluorescent edge around the rest of the 79 lesion suggesting transition to less active disease (Figure 3C-F).

80 Swept-source OCT (SS-OCT) (Triton $^{\circledR}$, Topcon Corporation, Tokyo, Japan) at the active 81 edge illustrated hyper-reflectivity of the ellipsoid zone (EZ), retinal pigment epithelium 82 (RPE) elevation and focal choroidal thickening (Figure 4A). Within areas of scarring, 83 irregularity of EZ, hyper-reflectivities within the RPE, choroidal attenuation and outer retinal 84 atrophy were observed (Figure 5A). SS-OCTA (Triton ${ }^{\circledR}$ ) segmentation images demonstrated 85 disrupted choriocapillaris vascular flow and hypoperfusion within the lesion corresponding 86 with hypocyanescent areas on ICGA. (Figure 6A and B) The deep and superficial vascular 87 plexus flow was normal.

88 Uveitis investigations were negative except for positive QuantiFERON ${ }^{\circledR}$-TB Gold and 89 tuberculin skin test (12 $\mathrm{mm}$ induration). The patient received anti-tuberculous therapy (ATT) 90 for 6-months alongside corticosteroid therapy (starting dose $80 \mathrm{mg} /$ day) tapered with clinical 91 response.

92 Over 9 months the lesions gradually became inactive: FAF demonstrated transition to an 93 inactive hypoautofluourescent lesion (Figure 2C-F); SS-OCT within the active area showed 94 resolution of RPE elevation, outer retinal atrophy and some restoration of outer retinal 
95 structures (Figure 4); OCTA revealed progressive improvement in choriocapillaris flow and

96 reduction in the size of the hypoperfusion areas suggestive of vascular reperfusion and

97 remodeling (Figure 6). The macula was spared, symptomatic improvement was reported and

98 excellent visual acuity maintained.

\section{Discussion}

100 Optical coherence tomography angiography (OCTA) is an emerging technology in the study

101 of inflammatory choriocapillaris diseases; it provides high-resolution structural information

102 at defined levels including the choriocapillaris, which may not be detectable on FFA or

103 ICGA. In TB-SLC, previous publications have described choriocapillaris hypoperfusion

104 within choroiditis lesions using OCTA at single time points, ${ }^{7,8}$ and correlated with enhanced

105 depth imaging OCT (EDI-OCT) structural change. ${ }^{6,7}$ Evidence on OCTA of paradoxical

106 worsening of choriocapillaris hypoperfusion following initiation of anti-tuberculous therapy

107 in 5 patients has been reported. ${ }^{9}$ Early OCTA detection of secondary choroidal neovascular

108 membrane (CNVM) is also described. ${ }^{8}$ Our case illustrated the use of OCTA for longitudinal

109 choriocapillaris flow evaluation, which has not been previously reported.

110

111 Four disease stages, characterised by FAF, have been described in TB-SLC - stage 1 (active):

112 diffuse hyperautofluorescence with an ill-defined hyperautofluorescent halo; stage 2

113 (subacute): defined surrounding edge of hypoautofluorescence; stage 3: predominant

114 hypoautofluorescence with stippled pattern and stage 4 (inactive): uniform

115 hypoautofluorescence. ${ }^{5}$ We studied SS-OCT, FAF and SS-OCTA during transition through

116 all disease stages. The correlation of these modalities at sequential disease stages, during

117 transition to inactive disease, has not been previously detailed. Progressive restoration of

118 choroidal circulation, with reduction of the lesion size and reduction in hypoperfusion was

119 seen on OCTA, suggesting vascular remodeling during the recovery phase. The 
120 choriocapillaris flow appeared to progressively restore centrally from the lesion edges. This

121 correlated with progressive change in FAF signal and transition to diffuse

122 hypoautofluorescence. At the last follow-up, persistent choriocapillaris flow abnormality was

123 evident and it is uncertain if the flow morphology will normalise with longer follow-up.

124 Interestingly in acute posterior multifocal placoid pigment epitheliopathy, choriocapillaris

125 healing is reported to lag behind resolution of outer-retinal morphological changes; ${ }^{10}$ and a

126 similar evolution may be observed in TB-SLC. Certainly, SS-OCT morphological

127 abnormalities including RPE elevation and hyper-reflectivity in the outer retina have evolved

128 within the active lesion ahead of full restoration of choriocapillaris flow.

130 Enhanced depth imaging (EDI-OCT), using spectral domain OCT for detailed choroidal 131 imaging, has been detailed in this condition. ${ }^{6,7}$ Rifkin et al demonstrated infiltration of the

132 choroid, elevation of RPE and focal choroidal thickening in the active lesion of TB-SLC 133 using EDI-OCT. ${ }^{6}$ We evaluated SS-OCT and found consistent imaging descriptors with focal 134 choroidal thickening and RPE elevation in acute phase and resolution in time. These EDI-

135 OCT features have not been reported in SC and may help differentiate infective SLC from 136 SC. $^{6}$ In a previous study, OCTA in the acute disease phase (stage 1 FAF lesions), areas of 137 reduced choriocapillaris flow within lesions correlated with reduced signal transmission on 138 EDI-OCT and the authors inferred that the observed flow void may be because of a 139 shadowing effect from hyper-reflective overlying retina. ${ }^{7}$ In our patient, we correlated OCTA 140 with SS-OCT at the active edge; hyper-reflective outer retinal changes were observed but 141 there was no loss of SS-OCT signal transmission and we believe this demonstrates true 142 hypoperfusion. 
144 These modalities, used in combination, provide valuable imaging markers of clinical activity

145 and disease evolution with the advantage of being non-invasive and readily repeatable during 146 follow up. Further studies to correlate structural change with function, quantitative analysis

147 of hypoperfusion and response to treatment are necessary.

148

149

150 References

151 1. Laatikainen L, Erkkilä H. Serpiginous choroiditis. Br J Ophthalmol 1974;58:777-783.

152 2. Gupta V, Gupta A, Arora S, Bambery P, Dogra MR, Agarwal A. Presumed tubercular

153 serpiginouslike choroiditis: clinical presentations and management. Ophthalmology $154 \quad 2003 ; 110: 1744-1749$.

155 3. Witmer R. A specific form of recidivating choroiditis. Ophthalmologica $156 \quad 1952 ; 123: 353-354$.

157 4. Schlaegel TF, O'Connor GR. Metastatic nonsuppurative uveitis. Int Ophthalmol Clin $158 \quad 1977 ; 17: 87-108$.

159 5. Gupta A, Bansal R, Gupta V, Sharma A. Fundus autofluorescence in serpiginous like 160 choroiditis. Retina 2012;32:814-25.

161 6. Rifkin LM, Munk MR, Baddar D, Goldstein DA. A new OCT finding in tuberculous 162 serpiginous-like choroidopathy. Ocul Immunol Inflamm 2015;23:53-58.

163 7. Mandadi SKR, Agarwal A, Aggarwal K, et al. Novel findings on optical coherence 164 tomography angiography in patients with tubercular serpiginous-like choroiditis. Retina $165 \quad 2017 ; 37: 1647-1659$.

166 8. Yee HY, Keane PA, Ho SL, Agrawal R. Optical coherence tomography angiography 167 of choroidal neovascularization associated with tuberculous serpiginous-like choroiditis. Ocul 168 Immunol Inflamm 2016;24:699-701. 
169 9. Agarwal A, Aggarwal K, Deokar A, et al. Optical coherence tomography angiography

170 features of paradoxical worsening in tubercular multifocal serpiginoid choroiditis. Ocul

171 Immunol Inflamm 2016;24(6):621-30.

172 10. Burke TR, Chu CJ, Salvatore S, et al. Application of OCT-angiography to characterise

173 the evolution of chorioretinal lesions in acute posterior multifocal placoid pigment

174 epitheliopathy. Eye (Lond) 2017;31(10):1399-408.

\section{Figure Legends}

176 Figure 1: Fundus photographs of a serpiginous-like choroiditis lesion contiguous with the

177 optic disc. (A) At 1 month the lesion has a light defined surrounding border (marked with

178 arrow). The inferior portion of the lesion is active with less well-defined borders and active

179 choroiditis (marked with *) representing contiguous extension. Progressive scarring is

180 evident during follow-up with no lesion extension at (B) 3 months; (C) 5 months (D); 6

181 months and (E) 9 months. The border has faded and progressive atrophy is observed. The

182 green arrows delineate the orientation of swept-source optical coherence tomography 183 (Figures 4 and 5).

184

185 Figure 2: Evolution of fundus autofluorescence over 9 months, from active progressive 186 disease to inactive state. The lesion was classified according to the pattern of 187 autofluourescence. ${ }^{5}$ (A) Baseline. Stage 1 'acute' lesion: defined, diffusely 188 hyperautofluorescent lesion with surrounding ill-defined hyperautofluoresecence. (B) 1 189 month. Stage 1-2 lesion: contiguous serpiginous-like extension compared to (A); the inferior 190 area has persistent hyperautofluoresecence and an ill-defined hyperautofluorescent border 191 (short arrows), indicating stage 1 active disease; a hypoautofluorescent thin border has 192 evolved around the rest of the lesion (arrow heads), a defining feature of transition to stage 2 193 'subacute' disease. (C) 3 months and (D) 5 months: progressive transformation, with stippled 
autofluorescence pattern and no lesion extension. (E) 8 months. Stage 3 lesion:

195 predominantly hypoautofluorescent lesion. (F) 9 months. Evolution towards stage 4, inactive

196 lesion, with transformation to uniform hypoautofluorescence within the lesion.

198 Figure 3: Multimodal imaging of the right eye. (A) Red-free imaging delineates hyper199 reflective choroiditis lesion. The inferior active edge is less well-defined than the remaining 200 border. (B) Fundus autofluorescence (FAF) image (see Figure 2B legend). (C), (D) Fundus

201 fluorescein angiography demonstrates different disease stages within the lesion: the active 202 inferior aspect shows early hypofluorescence due to blockage and progressive diffuse 203 staining and leakage indicative of active disease (marked with ${ }^{*}$ ) and the rest of the lesion 204 showed hypofluorescence and later edge hyperfluorescence (marked with an arrow) 205 correlating with the hypoautofluorescent thin border seen on FAF. (E and F) Early and late 206 indocyanine green angiography hypocyanescence was present, indicating choroidal disease.

Figure 4: Swept-source optical coherence tomography (SS-OCT) images orientated as per 209 Figure 1B at the active lesion edge. (A) At 1 month, SS-OCT within the advancing active area showed retinal pigment epithelium (RPE) elevation (arrow head), hyper-reflectivity of

211 ellipsoid zone (arrow) (EZ) and choroidal thickening. (B) At 3 months, SS-OCT showed 212 resolution of RPE elevation, persistent focal ellipsoid zone (EZ) hyper-reflectivity and 213 atrophy of the outer retina layers. (C) At 9 months, SS-OCT revealed resolution of EZ hyper214 reflectivity and partial restoration of outer retinal structures.

216 Figure 5: Swept-source optical coherence tomography OCT (SS-OCT) images orientated as 217 per Figure 1A within an area of scarring. (A) At 1 month, SS-OCT showed outer retinal 218 disruption including loss of the ellipsoid zone (EZ), hyper-reflectivities (marked with 
219 arrows), choroidal attenuation and outer retinal atrophy. This is contrasted by normal retinal 220 and choroidal appearance temporal to the optic disc. At 3 months (B) and 9 months (C), SS-

221 OCT showed persistent outer retinal atrophy but less hyper-reflectivity.

222

223 Figure 6: Sequential swept-source optical coherence tomography angiography (OCTA) 224 imaging suggests inflammatory vascular occlusive pathology in the acute stage with defined 225 areas of altered flow and non-perfusion at the level of the choriocapillaris that corresponded 226 with areas of hypocyanescence on indocyanine green angiography (A). (B) OCTA, at 1 227 month, revealed defined areas of choriocapillaris hypoperfusion within the lesion. (C) 3 228 months and (D) 9 months: Progressive OCTA evolution, over 9 months, with sequential 229 improvement in flow and reduction in size of the non-perfused areas was noted indicating 230 vascular remodelling in the choriocapillaris. 